Supplement of The Cryosphere, 11, 407-426, 2017

http://www.the-cryosphere.net/11/407/2017/

doi:10.5194/tc-11-407-2017-supplement

(C) Author(s) 2017. CC Attribution 3.0 License.

(c) (i)

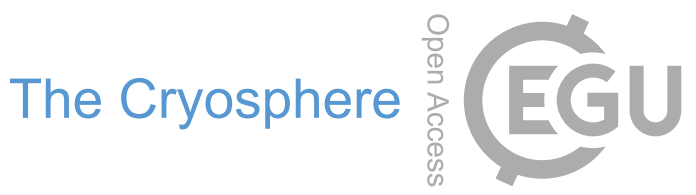

Supplement of

\title{
Spatial variability in mass loss of glaciers in the Everest region, central Himalayas, between 2000 and 2015
}

Owen King et al.

Correspondence to: Owen King (gy08ok@leeds.ac.uk)

The copyright of individual parts of the supplement might differ from the CC-BY 3.0 licence. 


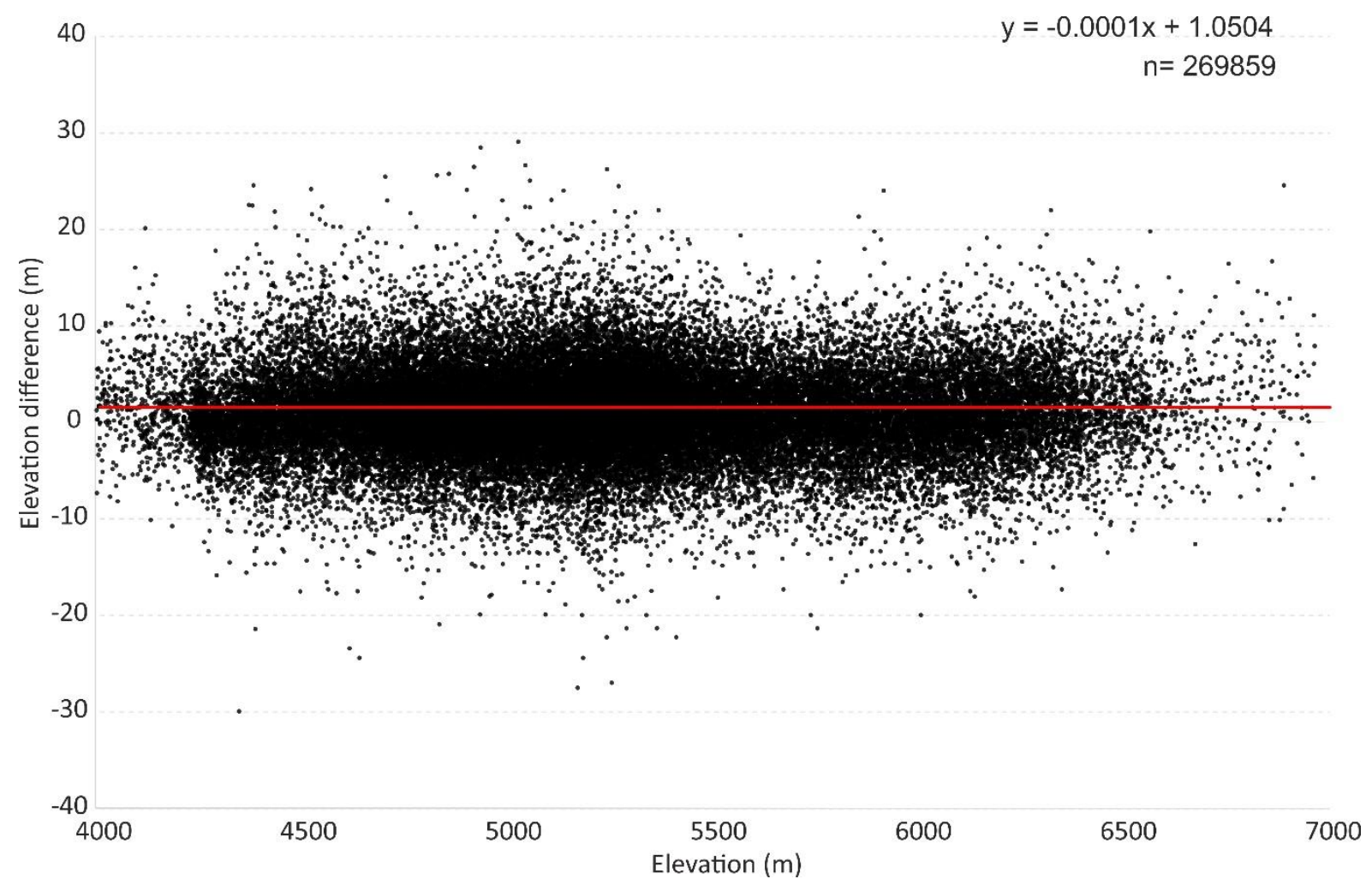

Figure S1. Elevation differences for stable ground (off-glacier) between SETSM and SRTM DEMs, plotted against elevation. There is no clear relationship between DEM differences and increasing/ decreasing elevation (often labelled an elevation dependent bias). 


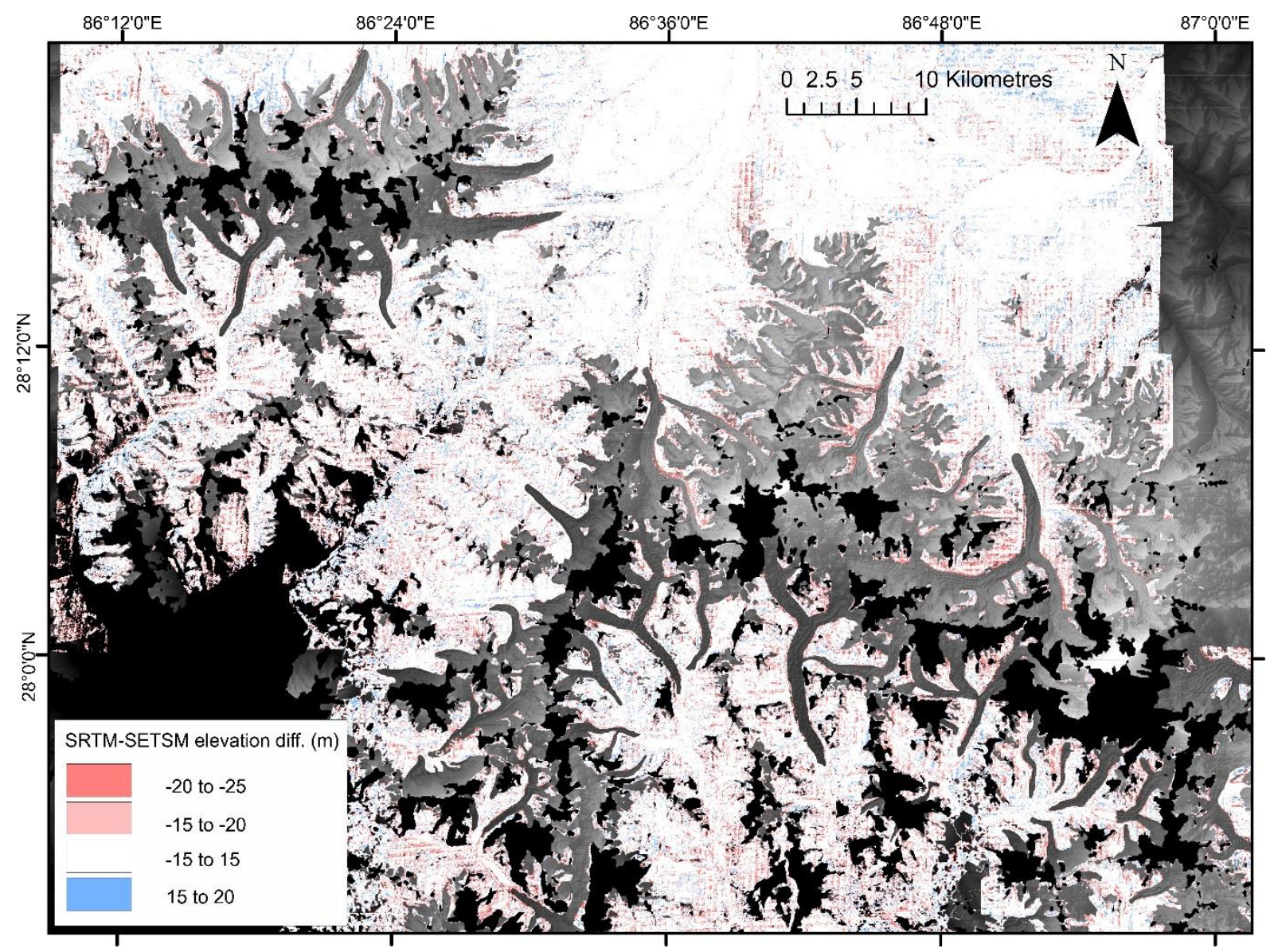

Figure S2. Elevation differences between the SRTM and SETSM DEMs over stable ground away from glacier surfaces. 
Table S1. Glaciers highlighted in the study. Data on glacier area and altitudinal range are taken from the GLIMS database. Glacier length is measured along centrelines from the bergschrund. Catchment notation: TK- Tama Koshi; DK- Dudh Koshi; P- Pumqu; P clean (surface debris-free) glaciers in the Pumqu catchment.

\begin{tabular}{|c|c|c|c|c|c|c|c|c|}
\hline \multirow[b]{2}{*}{ GLIMS ID } & \multirow[b]{2}{*}{ Name } & \multirow[b]{2}{*}{$\begin{array}{l}\text { Lengt } \\
\mathrm{h} \\
(\mathrm{km})\end{array}$} & \multirow[b]{2}{*}{$\begin{array}{l}\text { Area } \\
\left(\mathrm{km}^{2}\right)\end{array}$} & \multicolumn{3}{|c|}{ Altitude (m) } & \multirow[b]{2}{*}{ Lake status } & \multirow[b]{2}{*}{ Catchment } \\
\hline & & & & Min & Max & Range & & \\
\hline G086218E28282N & Bamolelingja & 7.4 & 15.4 & 5013 & 6745 & 1732 & No lake & TK \\
\hline G086280E28276N & G1 & 12.3 & 42.7 & 4778 & 7045 & 2467 & No lake & TK \\
\hline G086550E28133N & Yanong & 3.4 & 4.4 & 4984 & 6377 & 1393 & Proglacial lake & TK \\
\hline G086548E28174N & Yanong North & 3.2 & 4.1 & 5025 & 6524 & 1499 & Proglacial lake & TK \\
\hline G086384E28259N & Erbu & 10.9 & 25.8 & 5020 & 7130 & 2310 & No lake & TK \\
\hline G086471E27959N & $\begin{array}{l}\text { Drogpa } \\
\text { Nagtsang }\end{array}$ & 8.2 & 25.3 & 5018 & 7031 & 2013 & $\begin{array}{l}\text { Supraglacial } \\
\text { lake }\end{array}$ & TK \\
\hline G086537E27874N & Trakarding & 17.7 & 35.4 & 4561 & 6659 & 2098 & Proglacial lake & TK \\
\hline G086519E27919N & Ripimo Shar & 10.3 & 19.7 & 4600 & 6683 & 2083 & No lake & TK \\
\hline G086533E28088N & Shalong & 7.9 & 18.4 & 5301 & 6835 & 1534 & No lake & TK \\
\hline G086771E28015N & Ngozumpa & 22.2 & 80.7 & 4686 & 8176 & 3490 & $\begin{array}{c}\text { Supraglacial } \\
\text { lake }\end{array}$ & DK \\
\hline G086949E27913N & Imja & 6.5 & 15.3 & 5021 & 7998 & 2977 & $\begin{array}{c}\text { Supraglacial } \\
\text { lake }\end{array}$ & DK \\
\hline G086820E27978N & Khumbu & 15.7 & 39.5 & 4915 & 8062 & 3147 & $\begin{array}{l}\text { Coalescing } \\
\text { ponds }\end{array}$ & DK \\
\hline G086625E28029N & Lumbsamba & 9.1 & 12.5 & 4936 & 7258 & 2322 & No lake & DK \\
\hline G086917E27925N & Lhotse & 7.1 & 6.9 & 4821 & 6082 & 1261 & No lake & DK \\
\hline G086541E27988N & Melung & 6.5 & 7.6 & 5271 & 6028 & 757 & No lake & DK \\
\hline G086587E28039N & Bhote Kosi & 14.4 & 28.4 & 4793 & 6679 & 1886 & No lake & DK \\
\hline G086900E27843N & Hungu & 4.7 & 13.9 & 5207 & 6942 & 1735 & Proglacial lake & DK \\
\hline G086900E27843N & Marala & 2.9 & 13.9 & 5366 & 5920 & 554 & Proglacial lake & DK \\
\hline G086798E28111N & Jiuda & 10.1 & 15.9 & 5405 & 7801 & 2396 & No lake & $\mathrm{P}$ \\
\hline G086719E28132N & Gyachung & 13.6 & 47.1 & 5309 & 7853 & 2544 & No lake & $\mathrm{P}$ \\
\hline G086939E28060N & Rongbuk East & 10.9 & 26.7 & 5640 & 8361 & 2721 & No lake & $\mathrm{P}$ \\
\hline G086866E28050N & Rongbuk & 19.5 & 73.2 & 5153 & 8758 & 3605 & $\begin{array}{c}\text { Supraglacial } \\
\text { lake }\end{array}$ & $\mathrm{P}$ \\
\hline G086466E28321N & Ayi & 8.6 & 7.27 & 5313 & 6863 & 1550 & $\begin{array}{l}\text { Coalescing } \\
\text { ponds }\end{array}$ & $\mathrm{P}$ \\
\hline G086456E28291N & Tibet 1 & 12.9 & 26.8 & 5138 & 7085 & 1947 & $\begin{array}{l}\text { Coalescing } \\
\text { ponds }\end{array}$ & $\mathrm{P}$ \\
\hline G086633E28122N & Gyabrag & 11.5 & 33.2 & 5095 & 8182 & 3087 & No lake & $\mathrm{P}$ \\
\hline G086235E28330N & Longmojian & 4.5 & 9.3 & 5348 & 6788 & 1440 & Proglacial lake & $\mathrm{P}$ \\
\hline G086382E28331N & Duiya & 9.3 & 22.5 & 5480 & 7201 & 1721 & Proglacial lake & $\mathrm{P}$ \\
\hline G086395E28347N & Duosangpuxi & 5.5 & 7.7 & 5561 & 6992 & 1431 & No lake & $\mathrm{P}$ clean \\
\hline G086657E28179N & Siguang & 5.2 & 5.8 & 5652 & 6866 & 1214 & No lake & $\mathrm{P}$ clean \\
\hline G086423E28367N & $\begin{array}{l}\text { Duosangpudo } \\
\text { ng }\end{array}$ & 6.3 & 8.8 & 5502 & 6925 & 1423 & No lake & $\mathrm{P}$ clean \\
\hline G086709E28242N & G08 & 3.8 & 6.4 & 5726 & 6475 & 749 & No lake & $\mathrm{P}$ clean \\
\hline G086275E28322N & G06 & 6.0 & 6.0 & 5545 & 6926 & 1381 & No lake & $\mathrm{P}$ clean \\
\hline
\end{tabular}


Table S2. Mean and maximum surface lowering rates measured in glacier ablation zones, and geodetic mass balance estimates for each glacier included in the study. Bold text indicates lacustrine-terminating glaciers; means are italicised. The uncertainty of mass balance estimates contains an additional $7 \%$ error compared to the surface lowering estimates due to potential errors in the density conversion.

\begin{tabular}{|c|c|c|c|c|}
\hline Glacier & $\begin{array}{l}\text { Mean SL rate (m } \\
\left.\mathrm{a}^{-1}\right)\end{array}$ & $\begin{array}{l}\text { Maximum SL rate } \\
\left(\mathrm{m} \mathrm{a}^{-1}\right)\end{array}$ & $\begin{array}{l}\text { Mass balance (m } \\
\left.\text { w.e. } \mathrm{a}^{-1}\right)\end{array}$ & Catchment \\
\hline Bamolelingja & $-0.58 \pm 0.19$ & $-1.50 \pm 0.19$ & $-0.63 \pm 0.20$ & TK \\
\hline G1 & $-0.47 \pm 0.19$ & $-1.25 \pm 0.19$ & $-0.42 \pm 0.20$ & TK \\
\hline Erbu & $-0.50 \pm 0.13$ & $-0.73 \pm 0.13$ & $-0.32 \pm 0.14$ & TK \\
\hline Ripimo Shar & $-0.72 \pm 0.24$ & $-0.97 \pm 0.24$ & $-0.30 \pm 0.25$ & TK \\
\hline Shalong & $-0.52 \pm 0.10$ & $-0.96 \pm 0.10$ & $-0.32 \pm 0.11$ & TK \\
\hline Mean & $-0.55 \pm 0.17$ & $-1.08 \pm 0.17$ & $-0.40 \pm 0.18$ & \\
\hline Ngozumpa & $-0.64 \pm 0.18$ & $-1.17 \pm 0.18$ & $-0.53 \pm 0.19$ & DK \\
\hline Khumbu & $-0.84 \pm 0.14$ & $-1.34 \pm 0.14$ & $-0.35 \pm 0.15$ & DK \\
\hline Lumbsamba & $-0.31 \pm 0.15$ & $-0.89 \pm 0.15$ & $-0.17 \pm 0.16$ & DK \\
\hline Lhotse & $-0.66 \pm 0.13$ & $-0.99 \pm 0.13$ & $-0.65 \pm 0.14$ & DK \\
\hline Melung & $-0.40 \pm 0.11$ & $-1.12 \pm 0.11$ & $-0.74 \pm 0.12$ & DK \\
\hline Bhote Kosi & $-0.72 \pm 0.14$ & $-1.33 \pm 0.14$ & $-0.58 \pm 0.15$ & DK \\
\hline Mean & $-0.59 \pm 0.14$ & $-1.14 \pm 0.14$ & $-0.50 \pm 0.15$ & \\
\hline Jiuda & $-0.64 \pm 0.23$ & $-1.33 \pm 0.23$ & $-0.55 \pm 0.24$ & $\mathrm{P}$ \\
\hline Gyachung & $-0.66 \pm 0.29$ & $-1.07 \pm 0.29$ & $-0.44 \pm 0.31$ & $\mathrm{P}$ \\
\hline Rongbuk East & $-1.04 \pm 0.18$ & $-2.45 \pm 0.18$ & $-0.44 \pm 0.19$ & $\mathrm{P}$ \\
\hline Rongbuk & $-1.31 \pm 0.32$ & $-2.02 \pm 0.32$ & $-0.57 \pm 0.34$ & $\mathrm{P}$ \\
\hline Ayi & $-0.68 \pm 0.24$ & $-1.45 \pm 0.24$ & $-0.58 \pm 0.26$ & $\mathrm{P}$ \\
\hline Tibet 1 & $-1.17 \pm 0.20$ & $-2.14 \pm 0.20$ & $-0.83 \pm 0.21$ & $\mathrm{P}$ \\
\hline Gyabrag & $-1.22 \pm 0.22$ & $-3.40 \pm 0.22$ & $-0.74 \pm 0.23$ & $\mathrm{P}$ \\
\hline Mean & $-0.96 \pm 0.24$ & $-1.98 \pm 0.24$ & $-0.59 \pm 0.26$ & \\
\hline Duosangpuxi & $-0.53 \pm 0.20$ & $-1.30 \pm 0.20$ & $-0.24 \pm 0.26$ & P-clean \\
\hline Siguang & $-0.61 \pm 0.23$ & $-1.06 \pm 0.23$ & $-0.29 \pm 0.36$ & P-clean \\
\hline Duosangudong & $-0.31 \pm 0.24$ & $-0.91 \pm 0.24$ & $-0.29 \pm 0.16$ & P-clean \\
\hline G08 & $-0.49 \pm 0.21$ & $-0.96 \pm 0.21$ & $-0.21 \pm 0.36$ & P-clean \\
\hline G06 & $-0.51 \pm 0.22$ & $-0.98 \pm 0.22$ & $-0.24 \pm 0.16$ & P-clean \\
\hline Mean & $-0.49 \pm 0.22$ & $-1.04 \pm 0.22$ & $-0.25 \pm 0.24$ & \\
\hline Longmojian & $-1.12 \pm 0.21$ & $-2.53 \pm 0.21$ & $-0.91 \pm 0.22$ & $\mathbf{P}$ \\
\hline Duiya & $-0.65 \pm 0.12$ & $-1.45 \pm 0.12$ & $-0.45 \pm 0.13$ & $\mathbf{P}$ \\
\hline Hungu & $-0.54 \pm 0.25$ & $-1.10 \pm 0.25$ & $-0.56 \pm 0.27$ & DK \\
\hline Marala & $-0.61 \pm 0.30$ & $-2.24 \pm 0.30$ & $-0.88 \pm 0.32$ & DK \\
\hline Imja & $-0.84 \pm 0.22$ & $-1.62 \pm 0.22$ & $-0.79 \pm 0.24$ & DK \\
\hline Drogpa Nagtsang & $-0.78 \pm 0.33$ & $-1.91 \pm 0.33$ & $-0.75 \pm 0.35$ & TK \\
\hline Trakarding & $-0.94 \pm 0.39$ & $-2.19 \pm 0.39$ & $-0.48 \pm 0.41$ & TK \\
\hline Yanong & $-1.55 \pm 0.17$ & $-3.78 \pm 0.17$ & $-0.76 \pm 0.18$ & TK \\
\hline Yanong North & $-1.13 \pm 0.24$ & $-3.06 \pm 0.24$ & $-0.62 \pm 0.25$ & TK \\
\hline Mean & $-0.90 \pm 0.25$ & $-2.20 \pm 0.25$ & $-0.70 \pm 0.27$ & \\
\hline
\end{tabular}


Table S3. Hypsometric Index (HI) scores and classification, accumulation area ratio (AAR) and total area loss for each glacier included in the study. $\mathrm{EQ}=$ Equidimensional; $\mathrm{BH}=$ Bottom Heavy; $\mathrm{VBH}=$ Very Bottom Heavy; TH = Top Heavy. The ELA of the Lhotse and Melung glaciers are now above their altitudinal ranges, thus AARs cannot be calculated. Catchments: TK- Tama Koshi; DK- Dudh Koshi; TP- Tibetan Plateau. Lacustrine terminating glaciers are in bold; mean values are italicised.

\begin{tabular}{|c|c|c|c|c|c|c|}
\hline Glacier & $\begin{array}{l}\text { HI } \\
\text { score }\end{array}$ & $\begin{array}{l}\text { HI } \\
\text { classification }\end{array}$ & AAR & $\begin{array}{l}\text { Area change }\left(\mathrm{km}^{2}\right) \& \% \text { of } \\
\text { total area. }\end{array}$ & $\begin{array}{l}\text { Approximate } \\
\text { ELA }(\mathrm{m})\end{array}$ & Catchment \\
\hline Bamolelingja & 0.91 & EQ & 0.37 & $-0.07 \pm 0.08(0.44 \%)$ & 5593 & TK \\
\hline G1 & 1.45 & $\mathrm{BH}$ & 0.46 & $-0.49 \pm 0.22(1.15 \%)$ & 5659 & TK \\
\hline Yanong & 0.94 & EQ & 0.50 & $-\mathbf{0 . 3 1} \pm 0.02(6.33 \%)$ & 5557 & TK \\
\hline Yanong North & 1.22 & EQ & 0.43 & $-\mathbf{0 . 1 6} \pm 0.02(\mathbf{4 . 0 7 \%})$ & 5622 & TK \\
\hline Erbu & 1.15 & EQ & 0.45 & $-0.05 \pm 0.13(0.24 \%)$ & 5654 & TK \\
\hline Drogpa & 1.18 & EQ & 0.38 & $-2.37 \pm 0.13(9.12 \%)$ & 5623 & TK \\
\hline \multicolumn{7}{|l|}{ Nagtsang } \\
\hline Trakarding & 0.69 & EQ & 0.33 & $-\mathbf{0 . 3 5} \pm 0.18(\mathbf{0 . 9 8 \%})$ & 5708 & TK \\
\hline Ripimo Shar & 0.98 & EQ & 0.57 & $-0.04 \pm 0.10(0.20 \%)$ & 5408 & TK \\
\hline Shalong & 1.21 & $\mathrm{BH}$ & 0.41 & $-0.09 \pm 0.09(0.45 \%)$ & 5288 & TK \\
\hline Mean & 1.14 & $E Q$ & 0.43 & $-0.43 \pm 0.11(2.55 \%)$ & 5568 & \\
\hline Ngozumpa & 2.62 & $\mathrm{VBH}$ & 0.39 & $-0.13 \pm 0.16(0.16 \%)$ & 5815 & DK \\
\hline Imja & 5.93 & $\mathrm{VBH}$ & 0.50 & $-0.5 \pm 0.06(3.21 \%)$ & 5366 & DK \\
\hline Khumbu & 3.95 & VBH & 0.51 & $-0.06 \pm 0.18(0.22 \%)$ & 5568 & DK \\
\hline Lumbsamba & 3.11 & VBH & 0.40 & $-0.02 \pm 0.05(0.15 \%)$ & 5527 & DK \\
\hline Lhotse & 1.93 & VBH & 0.27 & $0 \pm 0.03(0 \%)$ & 5149 & DK \\
\hline Melung & 1.52 & VBH & 0.50 & $-0.20 \pm 0.03(2.55 \%)$ & 5270 & DK \\
\hline Bhote Kosi & 2.36 & $\mathrm{VBH}$ & 0.38 & $-0.13 \pm 0.12(0.51 \%)$ & 5310 & DK \\
\hline Hungu & 1.69 & VBH & 0.54 & $-0.13 \pm 0.06(1.53 \%)$ & 5687 & DK \\
\hline Marala & 0.56 & EQ & 0.20 & $-\mathbf{0 . 1 1} \pm 0.06(2.2 \%)$ & 5607 & DK \\
\hline Mean & 2.63 & $V B H$ & 0.41 & $-0.14 \pm 0.11(1.17 \%)$ & 5477 & \\
\hline Jiuda & 1.45 & $\mathrm{BH}$ & 0.35 & $-0.10 \pm 0.06(0.62 \%)$ & 6223 & $\mathrm{P}$ \\
\hline Gyachung & 1.85 & $\mathrm{VBH}$ & 0.36 & $-0.06 \pm 0.17(0.21 \%)$ & 6290 & $\mathrm{P}$ \\
\hline Rongbuk East & 2.58 & $\mathrm{VBH}$ & 0.44 & $-0.80 \pm 0.10(2.85 \%)$ & 6419 & $\mathrm{P}$ \\
\hline Rongbuk & 3.25 & VBH & 0.48 & $-0.14 \pm 0.27(0.16 \%)$ & 5923 & $\mathrm{P}$ \\
\hline Ayi & 3.00 & $\mathrm{VBH}$ & 0.30 & $-0.31 \pm 0.03(3.90 \%)$ & 5722 & $\mathrm{P}$ \\
\hline Tibet 1 & 2.80 & VBH & 0.54 & $-0.35 \pm 0.10(1.38 \%)$ & 5653 & $\mathrm{P}$ \\
\hline Gyabrag & 2.83 & $\mathrm{VBH}$ & 0.33 & $-1.17 \pm 0.12(3.32 \%)$ & 6059 & $\mathrm{P}$ \\
\hline Longmojian & 3.09 & VBH & 0.41 & $-\mathbf{0 . 4 4} \pm 0.03(\mathbf{4 . 2 8 \%})$ & 5795 & $\mathrm{P}$ \\
\hline Duiya & 1.39 & ВH & 0.17 & $-\mathbf{0 . 5 0} \pm 0.08(\mathbf{2 . 0 7 \%})$ & 6256 & $\mathrm{P}$ \\
\hline Mean & 2.47 & $V B H$ & 0.37 & $-0.39 \pm 0.11(1.91 \%)$ & 6037 & \\
\hline Duosangpuxi & 1.23 & $\mathrm{BH}$ & 0.40 & $-0.16 \pm 0.22(2.07 \%)$ & 6276 & P clean \\
\hline Siguang & 1.03 & EQ & 0.50 & $-0.06 \pm 0.16(1.03 \%)$ & 6259 & $\mathrm{P}$ clean \\
\hline Duosangpudong & 1.19 & EQ & 0.42 & $-0.10 \pm 0.15(1.13 \%)$ & 6213 & $\mathrm{P}$ clean \\
\hline G08 & 0.76 & EQ & 0.68 & $-0.07 \pm 0.18(1.09 \%)$ & 6100 & $\mathrm{P}$ clean \\
\hline G06 & 1.73 & $\mathrm{VBH}$ & 0.36 & $-0.08 \pm 0.07(1.25 \%)$ & 6235 & $\mathrm{P}$ clean \\
\hline Mean & 1.18 & $E Q$ & 0.47 & $-0.09 \pm 0.15(1.31 \%)$ & 6216 & \\
\hline
\end{tabular}

